\title{
L'espace hospitalier comme révélateur des pratiques professionnelles au sein des services de soin
}

Nathalie Mercier

\section{(2) OpenEdition Journals}

Édition électronique

URL : http://journals.openedition.org/communicationorganisation/2659

DOI : 10.4000/communicationorganisation.2659

ISSN : $1775-3546$

Éditeur

Presses universitaires de Bordeaux

Édition imprimée

Date de publication : 1 mai 2002

ISSN : 1168-5549

Référence électronique

Nathalie Mercier, "L'espace hospitalier comme révélateur des pratiques professionnelles au sein des services de soin », Communication et organisation [En ligne], 21 | 2002, mis en ligne le 27 mars 2012 consulté le 19 avril 2019. URL : http://journals.openedition.org/communicationorganisation/2659 DOI : 10.4000/communicationorganisation.2659

Ce document a été généré automatiquement le 19 avril 2019

(c) Presses universitaires de Bordeaux 


\title{
L'espace hospitalier comme révélateur des pratiques professionnelles au sein des services de soin
}

\author{
Nathalie Mercier
}

\section{Introduction}

1 Les résultats de l'étude de cas que nous nous proposons de commenter ici sont issus d'une thèse en sciences de l'information et de la communication ${ }^{1}$ portant sur les représentations de l'espace architectural hospitalier. Un des objectifs de ce travail de doctorat était d'étudier les représentations de l'espace des usagers avant et après un déménagement au sein d'une structure hospitalière, les représentations étant définies comme l'interprétation des images mentales construites à partir du réel, immédiatement perçu par les sens, imaginé ou mémorisé.

2 De cette recherche, nous retiendrons ici quelques observations concernant le lien entre organisation spatiale et fonctionnement des services. Nous avons constaté à de nombreuses reprises que les conditions spatiales sont l'expression d'une réalité sociale et qu'elles ont un impact réel sur la communication interindividuelle et sur les pratiques professionnelles. Les exemples que nous retiendrons concernent l'expression de la hiérarchie à travers l'espace, les effets du passage d'un service éclaté spatialement en plusieurs sous-parties à un espace commun et l'impact du cloisonnement de lieux initialement communs et partagés sur les représentations des usagers.

\section{Le contexte de l'étude}

3 La thèse qui nous permet de présenter ces résultats visait à vérifier si, pour une population relativement homogène de soignants, il existait des récurrences dans les représentations de l'espace rendant possible l'établissement d'un rapport fixe entre 
l'espace et ses représentations dans une culture donnée. Une réponse positive permettant de penser la constitution d'un code de lecture de l'espace. Nous supposions en effet, comme cela avait été suggéré par les travaux sur l'habitat pavillonnaire entrepris par $\mathrm{N}$. Haumont et H. Raymond ${ }^{2}$, qu'il existait un nombre fini de "variants» (terme choisi en référence à l'ouvrage de Roland Barthes intitulé Le système de la mode ${ }^{3}$ ) et qu'à chacun de ces variants étaient attachées des représentations communes. Nous désirions également savoir si les représentations variaient selon le statut hiérarchique des acteurs (aidesoignant, infirmier, médecin...), si elles dépendaient de leur spécialité et si un changement de lieu de travail était susceptible de révéler des disparités interindividuelles notables.

Pour valider nos hypothèses, nous avons choisi de travailler sur la population de trois services de l'hôpital de la Pitié Salpetrière, avant et après leur déménagement dans le bâtiment Babinski situé dans l'enceinte même de cet hôpital pavillonnaire. Les spécialités des services étudiés ont été sélectionnées de façon à recouvrir des pratiques médicales et des relations aux patients aussi variées que possible : un service de neuroradiologie, des blocs de neurochirurgie et des consultations d'ORL. Les représentations ont été étudiées à partir de la retranscription d'entretiens semi-directifs réalisés auprès des usagers des trois services, une trentaine au total. Chaque personne a été interviewée deux fois, une première fois dans le mois qui a précédé son déménagement et, une seconde, six mois après le changement de lieu.

Les résultats concernant l'existence d'un code de lecture permettant de décrypter le «langage silencieux » de l'espace dont parle E-T Hall ${ }^{4}$ ne seront pas discutés ici. Nous nous contenterons de signaler que l'analyse a mis en avant des récurrences dans le discours des interviewés. Les thèmes identifiés se sont révélés fonctionner en opposition (grand et petit, haut et bas, espace dominant et espace dominé...) ce qui nous a incité à privilégier un type de découpage structuraliste. Les oppositions signifiantes que nous avons repérées (variants topographiques, variants d'ambiance, variants d'être et variants d'appropriation) s'accompagnaient de signifiés en nombre limité et porteurs de valeurs relativement stables témoignant de représentations communes de l'espace. Les résultats obtenus étaient indépendants de la profession des usagers interviewés et de leurs lieux d'exercice ce qui confirmait la validité de nos hypothèses de départ.

Nous ne présenterons ici que quelques-uns des résultats issus de l'étude des représentations de l'espace des usagers de l'hôpital (aides-soignants, infirmiers, surveillants, médecins anesthésistes, médecins ORL, neurochirurgiens). Ils ont été choisis parce qu'ils sont représentatifs de l'influence de l'espace sur la communication interindividuelle et sur les pratiques professionnelles et parce qu'ils montrent comment l'espace intervient dans les jeux de pouvoir entre acteurs hospitaliers.

\section{L'expression de la hiérarchie}

7 L'analyse des représentations des soignants des trois services avant et après leur déménagement permet de faire état d'une expression spatiale commune de la hiérarchie hospitalière. Le pouvoir peut être signifié symboliquement grâce aux variants que nous avons identifiés ${ }^{5}$. Notre étude confirme l'existence, déjà souvent observée (G-N. Fischer, E.-T. Hall...) d'une sorte de norme non dite régissant l'attribution des espaces en fonction du rang professionnel de l'usager. 

permettant d'exprimer la hiérarchie (grand/petit, clair/sombre, ouvert/fermé...) sont affectées des valeurs positives ou négatives susceptibles d'exprimer symboliquement la place de l'individu dans la hiérarchie. Les conclusions auxquelles nous avons abouti rejoignent en tous points celles de notre DEA ${ }^{6}$ et celles de G.-N. Fischer ${ }^{7}$ concernant les valeurs attachées à l'espace dans des structures de travail de type bureau. Pour ce dernier, les paramètres spatiaux précédemment cités «en favorisant l'expression de l'identité personnelle, sont autant de symboles qui renseignent sur la valeur psychologique de la place occupée par l'individu en tant qu'elle affirme et renforce son identité ». M. Edelman ${ }^{8}$ en 1978, avait montré leur triple rôle : servir de support à la reconnaissance de la valeur personnelle d'un membre et de son pouvoir, indiquer aux membres extérieurs le rang et l'importance de la personne dans l'organisation et renforcer le poids de l'ordre social régissant cette organisation. paramètres que nous avons étudiés ne signifie pas que ces critères soient absolus. En effet, comme l'a montré E.-T. Hall ${ }^{10}$, en France comme aux USA, l'expression du rang hiérarchique est extériorisée à travers l'espace, et en particulier la surface, mais il n'en est pas de même dans d'autres pays comme la Grande-Bretagne ou le Japon, où seule l'appartenance procure une identité.

Dans le cas que nous avons étudié, chaque paramètre spatial semblait doté d'un certain poids permettant d'établir des équivalences entre les espaces et de pondérer certaines inadéquations à la norme non dite régissant leur attribution (le bureau d'un patron n'a 
pas de fenêtre mais ce manque est pallié par sa grande taille et sa position centrale; les secrétaires sont isolées, en périphérie du service mais elles sont "tranquilles" et échappent quelque peu à la surveillance des tiers...). Les acteurs semblent rassurés lorsqu'ils possèdent un lieu conforme à leurs attentes et à leur statut. Les revendications concernant l'espace sont apparemment moins le reflet de nécessités d'ordre fonctionnel ou ergonomique que l'expression d'un besoin de reconnaissance sur le plan social. Le fait que le poste hiérarchiquement le plus important dispose d'un grand bureau indépendamment des besoins réels de son occupant - n'est, pour ainsi dire jamais discuté. À l'inverse, le même avantage, revendiqué par des individus de grade moindre - même si leur demande est licite - est généralement refusé. Dans ce dernier cas, les besoins personnels sont généralement réduits par les tiers à des exigences narcissiques a priori illégitimes.

La norme sociale est tellement intégrée dans les esprits que les cas où le statut de l'occupant et son espace n'étaient pas en phase furent toujours mentionnés, que l'inadéquation soit en faveur ou en défaveur des occupants. Le «trop bon éclairage du bureau des secrétaires", la "trop grande taille du bureau des adjoints au chef de service ", "l'absence de fenêtre dans le bureau du patron »... furent dénoncés par les occupants d'autres lieux du service. Il fut aussi des cas où les "trop grandes surfaces " déclenchèrent des "autocritiques ». Une surveillante, par exemple, dit "avoir eu des scrupules au début parce que son bureau était trop beau... ».

L'espace apparaissant comme un reflet relativement fidèle de l'organisation hiérarchique hospitalière, on peut imaginer que la connaissance des règles d'attribution des locaux puisse permettre, si tant est que ces dernières soient transposables à d'autres domaines d'étude, de décoder sur les plans le système de répartition du pouvoir dans une entreprise donnée.

Cela dit, l'expression de la hiérarchie semble évoluer. En matière d'immeubles de bureaux par exemple, on voit s'amorcer de nouvelles tendances ${ }^{11}$. Des aménagements plus « égalitaires et plus fonctionnels » voient le jour du fait de la tendance à la mondialisation de l'économie. En effet, les employés d'une même entreprise, susceptibles d'être dispersés un peu partout dans le monde, tendent à ne plus être affectés de façon fixe et à temps plein à un espace donné. Ces nouveaux lieux de travail visent à offrir aux employés tout ce dont ils ont besoin pour exécuter leur tâche dans un lieu qui puisse être partagé avec d'autres individus.

Les mesures actuelles vont également dans le sens d'une «standardisation des critères d'affectation, notamment par le moyen du benchmarking qui consiste à comparer différentes activités dans le but de les standardiser, l'espace alloué faisant lui-même partie de cette standardisation ${ }^{12} »$. La technologie évoluée des communications et de l'informatique contribuerait, quant à elle, à donner plus d'autonomie à l'individu et l'empowerment ${ }^{a 13}$ qui l'accompagne passerait par le développement d'espaces d'équipe ainsi que par la création de lieux formels et informels de rencontre ${ }^{14}$. Au nombre des nouvelles formes d'aménagements on compte, par ailleurs, «le télétravail, le bureau virtuel, le travail à domicile et les bureaux satellites, ainsi que les espaces non territoriaux, tel que le bureau « just-in-time $»^{15}$ le poste « adresse libre $»^{16}$ et l'« hotelling $»^{17} »$. 


\section{Changement de lieu, changement de pratiques}

Après avoir rendu compte de la traduction spatiale de la hiérarchie, nous nous proposons de montrer comment le changement d'espace est susceptible de remettre en cause les liens sociaux et professionnels. L'exemple du secteur «blocs opératoires » du service de neurochirurgie nous a permis de découvrir les effets du regroupement de lieux initialement dispersés sur les relations interindividuelles et le mode d'appropriation de l'espace

\section{État des lieux}

Pour comprendre la situation, nous ne pouvons faire l'économie d'un petit descriptif du service de neurochirurgie 1 de l'hôpital Pitié-Salpétrière. Ce dernier se décompose en trois secteurs, chacun voué à une activité particulière: le secteur bloc opératoire, le secteur consultation et le secteur hospitalisation. Avant son déménagement, le secteur bloc opératoire était composé de trois unités comprenant chacune une ou plusieurs salles d'intervention, une salle d'anesthésie, des vestiaires et une salle de stérilisation. Ces trois unités étaient, de l'avis des soignants, «éclatées aux quatre coins du bâtiment ». Celle que nous avons étudiée plus particulièrement était située à proximité du service de réanimation, d'une unité d'hospitalisation et de quelques salles de consultation. Après le déménagement, l'ensemble du service fut dispatché sur trois plateaux situés au R-l (secteur bloc), en mezzanine (secteur consultation) et au $\mathrm{R}+3$ (secteur hospitalisation). Le secteur bloc regroupa les équipes chirurgicales des trois unités précédemment décrites ainsi que celles relevant de trois autres services (neurochirurgie 2, ORL et ophtalmologie) dans un espace contenant au total neuf salles d'intervention juxtaposées disposant chacune d'une salle d'anesthésie attenante.

\section{D'une organisation familiale et autarcique...}

La dispersion des blocs opératoires de neurochirurgie telle qu'elle s'est présentée à nous initialement se soldait par un certain isolement des chirurgiens dénoncé par l'un d'eux comme « une aberration car lorsqu'un malade a un problème, on ne peut attendre de l'aide de personne ». Isolés les uns des autres, les neurochirurgiens se devaient donc d'être très autonomes car il leur était difficile de faire intervenir rapidement un confrère situé hors de l'unité.

31 La dispersion avait, par ailleurs, des répercussions négatives sur le plan logistique. Induisant des besoins similaires dans chaque bloc elle s'accompagnait de ce que certains décrivaient comme « un gaspillage en personnel et en matériel ».

En contrepartie, cet éclatement des structures eut des conséquences positives sur le plan affectif. Il permit d'atténuer la virulence des conflits entre certains soignants en donnant au surveillant la possibilité d'isoler les unes des autres les personnalités incompatibles. $\mathrm{Au}$ stade où nous l'avons observée, c'est à dire, après plusieurs mois, voire plusieurs années d'occupation par les mêmes soignants, nous avons constaté que l'espace favorisait au sein de chaque unité la constitution de sortes de "familles", selon l'expression retenue par l'un des occupants. Chaque famille comptant une dizaine de membres de différents statuts (aides-soignants, infirmiers, chirurgiens, anesthésistes). La 
fréquentation répétée des mêmes collègues et des mêmes lieux et la participation aux mêmes interventions neurochirurgicales renforcèrent la solidarité entre les membres de l'équipe et le sentiment d'appartenance à l'unité plus qu'au secteur. Cet attachement se traduisit verbalement, les paramédicaux évoquant leur «chez soi », certains disant être "perdus » à chaque changement de bloc. L'ambiance était décrite comme familiale et conviviale. « Les panseuses avaient mis des rideaux aux fenêtres et leurs pantoufles » (un neurochirurgien).

$\mathrm{Au}$ sein des groupes, la tendance était au nivellement des différences hiérarchiques (chacun étant considéré moins en fonction de son rang qu'en fonction de ses compétences et de sa personnalité) et à l'élargissement du champ des compétences professionnelles. Aides-soignantes et infirmières se voyaient confier des tâches qui outrepassaient leurs fonctions initiales du fait de la relation de confiance qui s'était construite entre les membres du personnel de l'équipe.

Nous avons par ailleurs constaté, au sein de cet espace, le développement de pratiques transgressées contribuant à renforcer les liens de complicité au sein du groupe et témoignant d'une appropriation certaine du territoire. La transgression se traduisait ici par un non-respect de certaines règles d'hygiène (séparation du linge propre et sale dans le même sas, conservation de la même tenue lors du passage de la porte du bloc.) et par des « détournements » de fonction, la salle de stérilisation étant parfois utilisée comme salle de repos ou office (il arrivait que « des soignants y installent une petite table pour y déjeuner le midi ou y prendre un café, y glandent, y discutent tranquillement d'un film ou $y$ fument une cigarette »).

Si la constitution des familles satisfaisait la plupart, elle déplaisait en revanche aux membres du personnel chargé de la gestion du service. Le surveillant notamment, se plaignait de ce que l'éclatement du bloc en trois sous-parties favorise l'apparition de « clans » repliés sur eux-mêmes, communiquant peu entre eux et refusant d'interchanger leurs membres. La résistance des soignants aux rotations étant probablement liée au fait que le changement de territoire aurait été susceptible de remettre en question l'équilibre acquis au sein de ces "forteresses matérielles et affectives». Le surveillant disait également éprouver des difficultés à surveiller le fonctionnement de ses équipes du fait de leur autonomisation et des distances spatiales séparant les trois unités. En contribuant à favoriser le développement de pouvoirs locaux et à mettre en échec l'autorité hiérarchique, l'espace agissait donc ici de façon coercitive.

\section{à un système plus « industriel » et hiérarchique}

36 Comme nous Pavons décrit, le déménagement eut pour effet de segmenter les trois secteurs du service sur trois niveaux différents et de regrouper les trois unités du secteur bloc initialement décrit dans un nouveau secteur comptant neuf salles d'intervention.

L'évocation du déménagement et l'idée d'avoir à partager un territoire avec d'autres services avait suscité des craintes chez les usagers qui avaient anticipé, semble-t-il, les conséquences que ces mesures pouvaient avoir sur les conditions d'exercice du pouvoir, sur la qualité des liens inter-individuels et sur le mode d'exercice de leur profession. Ils appuyaient leurs arguments sur le risque que les changements avaient de modifier leurs pratiques professionnelles et leurs rythmes de travail. Leurs craintes semblent avoir été en partie vérifiées. 

tentative de mécanisation et de rentabilisation du système de soins. Ils se sont sentis pris dans une logique visant à les utiliser pour des missions très spécifiques et ignorant leurs désirs. "On est déplacé comme des pions », rapporta l'un d'eux, «tout est conçu pour qu'on ne fasse que travailler et faire tourner tout le temps le plateau technique » dit un autre, « il n'y pas de lieu où attendre avant les interventions, hormis le couloir » ajouta un dernier. Les usagers se sentirent réduits au statut de producteurs de soins et non considérés en tant qu'individus ayant des besoins affectifs et sociaux spécifiques. secteur bloc contribuèrent à entraver le processus d'appropriation des nouveaux locaux. Six mois après le changement de lieu, le deuil de la situation précédente et des avantages qui y étaient attachés n'était pas fait. Les soignants continuaient à se plaindre de la nouvelle situation spatiale.

Les gestionnaires du service (patron du service et surveillant infirmier du bloc) ont été les seuls interviewés à exprimer leur contentement après le déménagement. Le changement de lieu leur permis de dissoudre les groupes rebelles aux rotations et d'imposer un fonctionnement en pool dans des salles d'intervention non fixes. Il leur permit également de surveiller plus aisément l'ensemble de leurs subalternes. L'espace eu donc un effet beaucoup plus opérant que les imprécations verbales. Le surveillant apprécia, par ailleurs, particulièrement le fait de pouvoir rencontrer quotidiennement des surveillants 
d'autres blocs car cela lui permettait de partager avec eux son expérience, ce qui ne lui était pas possible dans les locaux précédents, très éloignés de ceux des autres services (ORL, ophtalmologie...).

\section{Segmentation spatiale et cloisonnement intellectuel}

44 Si l'exemple précédent portait plus spécifiquement sur l'impact des transformations spatiales sur les relations interindividuelles au sein d'une équipe, celui que nous nous proposons d'exposer ici s'intéresse plus à montrer en quoi les modifications de l'organisation spatiale eurent un effet sur les pratiques professionnelles à travers les modifications des distances interindividuelles notamment.

\section{État des lieux}

Les locaux dont nous parlerons ici sont destinés à l'exercice des fonctions « administratives » de la consultation d'ORL. Dans l'ancien bâtiment, ils étaient regroupés dans un espace très étroit, une sorte de couloir, séparé de la salle d'attente par un guichet fermé à mi-hauteur par une grande vitre transparente. $Y$ étaient juxtaposées sans cloisonnement : l'accueil des consultants, le secrétariat, le bureau des rendez-vous et les archives. Cet espace susceptible de contenir quatre personnes était considéré comme « petit » par ses occupants.

Après le déménagement, ces locaux se sont retrouvés séparés les uns des autres en autant de pièces indépendantes affectées chacune à une tâche bien définie. Chacune de ces pièces pouvait être close excepté l'accueil séparé de l'entrée par une banque. Les nouveaux espaces abritaient des fonctions uniques et étaient de dimensions plus importantes que ceux qui étaient affectés aux mêmes fonctions précédemment. Le déménagement eu donc pour effet de segmenter les espaces et de modifier les distances interindividuelles.

\section{Des espaces ouverts de la promiscuité...}

Les espaces « administratifs » du premier bâtiment avaient un taux d'occupation élevé et contenaient un nombre d'objets important. Cette situation spatiale induisait un état de proximité physique, visuelle, olfactive et sonore tel qu'il permettait de parler d'une situation de promiscuité.

48 L'analyse des entretiens a révélé que cette promiscuité avait des répercussions négatives sur les déplacements des occupants, leurs possibilités de choix en matière d'aménagement et leur confort. En limitant leur liberté d'action, elle constituait un obstacle à l'appropriation du lieu.

Le partage d'un même local exerçait, par ailleurs, une influence péjorative sur la nature des communications entre les membres du personnel de l'équipe et entre ces derniers et « les autres » (soignants, patients...). Le bruit notamment nuisait à la qualité des échanges directs ou indirects (via le téléphone) et la proximité spatiale et auditive favorisait certaines indiscrétions (le secret médical était souvent bafoué). Les locaux interdisant toute confidentialité, les relations avaient tendance à être moins personnalisées. 
50 Du fait des nuisances sonores associées, la proximité physique eut également tendance à augmenter la fatigue et la charge de travail en rendant plus difficile la concentration sur une tâche. Les usagers d'un même lieu se plaignaient souvent de "difficultés à s'adapter », de « stress », de « confinement ». Ils décrivaient leur espace de travail comme centrifuge.

51 Cette promiscuité présenta toutefois quelques avantages puisqu'elle rendit incontournables les échanges interindividuels et le partage du savoir (du moins lorsque les tâches à effectuer n'étaient pas trop complexes). Elle favorisa la flexibilité et la polyvalence des soignants et leurs aptitudes à se remplacer les uns les autres. Comme dans le cas précédemment exposé, l'affectation fixe à un lieu, le côtoiement quotidien des mêmes collègues et les faibles distances interindividuelles contribuèrent par ailleurs à renforcer le sentiment d'appartenance à une communauté et le développement d'une certaine solidarité entre les membres de l'équipe.

\section{aux espaces cloisonnés et monofontionnels}

Le passage de territoires partagés à des territoires individuels fut ressenti positivement par les soignants. Il favorisa l'appropriation, chacun étant en mesure d'organiser relativement librement son nouvel espace de travail. La satisfaction de l'occupant était d'autant plus importante que le lieu attribué possédait des attributs spatiaux auxquels étaient conférées des valeurs positives (modernité, grande taille, éclairage naturel...) (cf. chapitre sur l'expression spatiale de la hiérarchie).

La nouvelle organisation spatiale permit aussi aux soignants de se soustraire au regard et à l'écoute de leurs voisins et d'échapper à leur surveillance. Mais cette indépendance eut un revers : elle obligea les soignants à prévenir leurs collègues lorsqu'ils quittaient leurs postes et à limiter leur temps d'absence au minimum, personne n'étant en mesure de les remplacer « au pied levé » comme cela se faisait auparavant, lorsque le voisinage était immédiat. Les déplacements furent donc moins libres et nécessitèrent plus d'efforts logistiques.

54 La segmentation des espaces eut, par ailleurs, des effets négatifs sur les compétences professionnelles. Les tâches affectées aux salariés dans les lieux cloisonnés devenant mieux définies que dans les espaces partagés, chacun eut désormais un poste bien identifié et une mission fixe mais son domaine de compétence diminua. Les postes n'étant plus juxtaposés au sein d'un même espace, les salariés ne furent plus spontanément au courant des activités de leurs collègues. Moins informés, moins sollicités, ils devinrent moins polyvalents, l'organisation d'ensemble fut «rigidifiée». Les individus devenant beaucoup moins substituables d'un poste à l'autre, les besoins en personnel augmentèrent.

55 La stricte définition des tâches attribuées à chaque poste de travail fut vécue négativement par ceux qui étaient psychiquement plus mobiles et plus enclins à apprendre et à élargir leurs savoirs. Elle fut en revanche vécue positivement par ceux qui n'aimaient pas être sollicités à propos de tâches ne relevant pas de leur strict domaine de compétences, probablement parce que cette situation supposait un effort d'adaptation et qu'elle était susceptible de les mettre en situation d'échec s'ils n'étaient pas en mesure de répondre à la demande. 
56 La surveillante tenta de contrebalancer la spécificité des tâches et l'immobilisme physique relatif favorisé par le cloisonnement des locaux par l'instauration arbitraire d'une rotation des postes. Mais cette décision, comme celle prise pour des raisons comparables par les supérieurs hiérarchiques du bloc opératoire de neurochirurgie (cf. exemple précédent), fut mal reçue par ses subalternes. Les raisons non dites des refus d'obtempérer étaient probablement liés, comme dans le cas précédent, au coût psychique d'un changement de poste et au changement de lieu d'exercice qui supposait un renoncement aux prérogatives en cours et une remise en cause des territoires appropriés.

D’une façon générale, on pourrait résumer la situation comme suit : les espaces assurant une certaine promiscuité sont propices aux échanges sur le plan professionnel, à une certaine polyvalence et à une certaine mobilité physique (possibilité de quitter son poste) et psychique mais ils sont ressentis comme fatigants et stressants. À l'inverse, les espaces cloisonnés permettant d'isoler les usagers les uns des autres tendent à favoriser l'autonomie des salariés et leur sens des responsabilités mais contribuent à leur immobilisation tant physique que psychique.

La promiscuité retentit sur la nature des échanges interindividuels dans la mesure où elle favorise les rencontres mais où la présence de tiers nuit à la confidentialité des relations. Le cloisonnement est, à l'inverse, propice à la confidentialité des échanges mais ne favorise pas les rencontres spontanées. Il diminue, de ce fait, le sens de la communauté et de l'entraide chez les occupants d'un service donné.

Des études opposant espaces partagés et non-partagés ont été menées dans d'autres domaines du tertiaire. Elles concernent pour l'essentiel l'opposition entre bureaux cloisonnés et open space ${ }^{18}$. Elles mettent en évidence le fait que les espaces ouverts sont invariablement évalués plus négativement que les espaces fermés qui assurent une certaine "privacité ». Ils sont jugés de façon péjorative sur le plan des préférences individuelles (G.-N. Fischer ${ }^{19}$ et A.-O. Dean) ${ }^{20}$, de la satisfaction et de la motivation des employés (G.-R. Oldham et D.-J. Brass) ${ }^{21}$, de leur rendement (A. Crouch et J. Nimran) ${ }^{22}$, de la qualité de leurs relations avec leurs collègues de travail (G.-R. Oldham et D.-J. Brass) ${ }^{23}$ et de la reconnaissance de leur statut de cadre (E. Sundstrom, R.-E. Burt et D. Kamp) ${ }^{24}$.

Longtemps on a pensé que la communication était associée à l'ouverture de l'espace ${ }^{25}$ et à la visibilité qui s'ensuivait et que le bureau paysager facilitait la circulation des interactions et le partage d'informations significatives et pouvait jouer un rôle facilitateur dans le règlement des conflits interpersonnels. En réalité, il n'en est rien. G.$\mathrm{N}$. Fischer ${ }^{26}$ souligne qu'aucune recherche n'a pu clairement mettre en évidence l'existence d'un lien entre ouverture de l'espace et meilleure communication, ni entre meilleure communication et augmentation du rendement d'ailleurs. M.-J. Hatch ${ }^{27}$, par exemple, démontre que la « privacité » contribue à accroître la communication alors que l'absence de " privacité » tend à la réduire.

61 Ces résultats corroborent pour partie ce que nous avons observé. Ils confirment l'intérêt des lieux isolés pour l'appropriation et l'impact négatif des lieux partagés sur la communication avec autrui mais ils diffèrent pour ce qui concerne les échanges professionnels. Le partage de locaux favorise ici le passage de l'information et l'adaptabilité des postes dans la mesure où ces derniers ne nécessitent pas de savoirs très spécifiques. 


\section{Conclusion}

62 L'étude des représentations des usagers nous a permis, à l'occasion d'un déménagement, de mieux appréhender le lien entre pratiques spatiales et pratiques sociales au sein de l'hôpital. Le cas que nous avons analysé dévoile les modalités d'expression spatiale de la hiérarchie et permet d'apprécier combien la règle non dite de répartition des surfaces, lumières, vues, densités... est intégrée par les usagers et leur permet de se situer socialement les uns par rapport aux autres.

Ce travail a eu également l'intérêt de montrer l'impact de l'espace sur le fonctionnement d'un service et, plus particulièrement, sur la gestion du personnel et l'exercice du pouvoir. Au bloc opératoire de neurochirurgie, par exemple, le passage de petits groupes structurés autour de trois salles d'opération non contiguës à un espace commun regroupant neuf salles d'opération fit éclater la cohésion des équipes transplantées et induisit une recomposition totale des alliances entre membres d'un même service. On est passé de liens reposants moins sur la hiérarchie que sur la complicité et l'entraide à la création de groupes s'organisant autour des statuts socioprofessionnels. Dans ce service, le changement d'espace a, par ailleurs, permis à la direction d'obtenir la rotation du personnel entre les différents blocs, de favoriser le processus de surveillance des équipes de soins et de créer les conditions propres à une économie de matériel humain et technique.

En ORL, le déménagement a également contribué à modifier les pratiques professionnelles de façon relativement déterminante. Les locaux "petits", ouverts et partagés du bâtiment initial induisaient une certaine promiscuité mais favorisaient le passage de l'information, le développement d'une relative pluridisciplinarité et l'entraide. Le déménagement dans des lieux plus vastes et segmentés dans lesquels le confort était supérieur à celui des espaces précédents, modifia les conditions de la communication. Elle devint moins spontanée. Le cloisonnement de l'espace s'accompagna d'un cloisonnement des savoirs et d'une tendance marquée à l'individualisme.

L'espace étant un des traducteurs de l'ordre social au sein de l'entreprise hospitalière et ayant des répercussions importantes sur les relations interindividuelles, l'exercice du pouvoir, les pratiques professionnelles et le sentiment d'appartenance au service, les conclusions auxquelles nous sommes parvenus nous semblent susceptibles d'être utiles à ceux qui sont impliqués dans la conception de nouveaux locaux et dans la gestion et le fonctionnement d'une organisation.

\section{NOTES}

1. Mercier N., Représentations de l'espace architectural hospitalier et communication entre les acteurs, Paris, thèse en SIC, Paris IV (Celsa), 2000.

2. Haumont N.. Raymond H., Les pavillonnaires : étude psychosociologique d'une mode d'habitat, Paris, éd. CRU, 1966. 
3. Barthes R., Le système de la mode, Paris, éd. du Seuil, 1967.

4. Hall E.-T., La dimension cachée, Paris, éd. du Seuil, 1971 ( $\mathrm{I}^{\mathrm{e}}$ ed. 1966).

5. Parmi les variants les plus signifiants nous retiendrons :

-des variants topographiques: variant de grandeur, variant d'altitude, variant de positionnement, variant de clôture et de transparence, variant d'assemblage,

-des variants d'ambiance : variant d'éclairage, variant chromatique, variant

chronologique, variant hygiène,

-un variant d'être : les objets techniques,

-des variants d'appropriation: variant de maitrise, variant de mobilité, variant de personnalisation, variant de distribution, variant de contrôle.

6. Mercier N., Les espaces architecturaux d'entreprise et la communication, DEA en SIC (Celsa), 1992.

7. Fischer G.-N., Vischer J., Évaluation des environnements de travail, 1997, p. 20.

8. Edelman M., Space and social order, Madison : Institue for Research on Poverty, University of Wisconsin, 1978, cité par Fischer G.-N., Vischer J., 1997, ibid. p. 68.

9. Fischer G.-N., Vischer J., Évaluation des environnements de travail, 1997, p. 27

10. Hall E.-T., Au-delà de la culture - Contexte et signification. 1979, p. 91.

11. Fischer G.-N.. Vischer J., 1997, op. cit. p. 90.

12. Fischer G.-N.. Vischer J., 1997, op. cit. p. 26.

13. «Il s'agit d'un concept appliqué aux façons contemporaines de gérer les entreprises où le travail est organisé pour responsabiliser les employés et pour assurer plus d'autonomie quant à la performance de leurs tâches ». Fischer G.-N., Vischer J., 1997, op. cit. p. 242.

14. Fischer G.-N., Vischer J., 1997, op. cit. p. 90.

15. «Postes de travail aménagés afin d'accommoder celui qui en a besoin pour des périodes de temps qui conviennent aux exigences de son travail ». Fischer G.-N., Vischer J., 1997, op. cil. p. 242. 16. "Aménagement de bureau où tous les postes sont ouverts à tous les membres d'une équipe de travail, selon leurs besoins et les exigences de leurs tâches ». Fischer G.-N., Vischer J., 1997, op. cit. p. 241.

17. «Locaux aménagés selon les principes d'adresse libre (free adress) mais où les bureaux doivent être réservés à l'avance et où un personnel de soutien est disponible en tout temps ". Fischer G.-N., Vischer J., 1997, op. cit.. p. 243.

18. Les open space sont apparus dans les années 60 . Le terme désigne un système de bureau aménagé en aires ouvertes, réparties sur le principe de création de zones de travail identifiées par des délimitations telles que les plantes vertes qui forment un paysage intérieur, d'où le terme de bureau-paysager qui en est la traduction française (Définition tirée de Fischer G.-N., Vischer J., L'évaluation des environnements de travail. 1997. p. 244.

19. En France, la majorité des employés expriment leur préférence pour le bureau individuel, éventuellement partagé à deux. Fischer G.-N.. Vischer J., 1997, ibid. p.24.

20. A.-O. Dean a montré qu'après quatre ans d'occupation de bureaux paysagers, deux tiers des employés disaient préférer un bureau personnel et fermé. Dean A.-O., Evaluation of an open office landscape : AIA Headquarters In American institute of architects journal, 66, 1977, pp. 32 39, Cité par Fischer G.-N., 1997, ibid. p. 77.

21. Oldham G.-R.. Brass D.-J.. Employee reaction to an open plan office: A naturally-occuring Quasiexpériement. in Administrative Science Ouartely_24, 1979. pp. 267-284. cité par Fischer G.-N. Vischer J., 1997, ibid. p. 25.

22. I1 existe une relation négative entre le nombre de personnes dans un espace ouvert et le rendement. Crouch A., Nimran J., Office Design and the Behavior of senior manager, in Human Relations, 42 (2). 1989, pp. 139.155.- Cité par Fischer G.-N., Vischer J., 1997, ibid. p. 24.

23. Oldham G.-R.. Brass D.-J.. 1979, ibid.

24. L'étude de E. Sundstrom, R-E. Burt et D. Kamp suggère que les cadres préfèrent un espace de travail personnel à un espace accessible ce qui indique une corrélation positive non seulement 
entre « privacité » et satisfaction mais aussi entre " privacité » et statut. Sundstrom (E.), Burt (RE.) et Kamp (D.), Privacity at work: Architectural correates of job satisfaction and job performance in Academy of management journal, 23 (1), 1980. pp. 101-117, cité par Fischer (G.-N.) et Vischer(J.), 1997, ibid. p. 77.

25. Le contact visuel entre les individus apparaissait à Sundrom et Bronron (1982) comme un facteur facilitant la communication. Fischer G.-N., et Vischer J., 1997, ibid. p. 39.

26. Fischer G.-N. et Vischer J., 1997, ibid. p. 40.

27. M-J. Hatch a montré, en comparant la valeur de la " privacité » dans un système ouvert ou fermé que les barrières physiques du bureau sont positivement associées au degré d'interaction et également au temps utilisé à travailler seul, ceci, tant pour les emplois techniques que professionnels. La «privacité » contribuerait donc à accroître la communication alors que l'absence de "privacité » tend à la réduire. Hatch M-J., Physical Barries, task characteristic and interaction activity in research and development firms in Administrative Science Quartely, 1987, pp. 387-399, cité par Fischer G.-N. et Vischer J.. 1997, ibid. p. 77.

\section{RÉSUMÉS}

Les résultats que nous exposons dans cet article font suite aux travaux menés lors d'une thèse portant sur les représentations de l'espace architectural hospitalier et la communication entre les acteurs du projet. Notre étude visait à comprendre les effets du déménagement de services hospitaliers sur les soignants et leurs représentations mentales de l'espace de travail. Elle nous a permis d'apprécier les effets du regroupement de lieux initialement dispersés et ceux de la segmentation de locaux initialement partagés sur les relations interindividuelles, l'exercice professionnel et les modalités d'appropriation de l'espace. L'étude révèle également comment l'espace traduit l'existence d'une hiérarchie hospitalière.

The deductions that we expose in this article are the continuation of a doctorate study based on the representations of architectural space in hospitals and the communication between the different actors within this context. The study aimed to understand the effects of relocating different services within hospitals, and the reaction and mental representations created by nursing staff due to their new work space. This study has resulted in a belter appreciation of the effects in bringing together various work spaces that were orinally dispersed, and the segmentation of others that were originally shared due to relations between staff, professional needs and the modalities associated with the appropriation of space. The study also revealed how the occupation of space can translate the hierarcy.

\section{INDEX}

Mots-clés : espace hospitalier, déménagement, appropriation, hiérarchie, interaction, lien social 


\section{AUTEUR}

\section{NATHALIE MERCIER}

Nathalie Mercier est docteur en médecine, docteur en sciences de l'information et de la communication et architecte DPLG. Enseignante en sociologie à l'école d'architecture Paris Belleville et journaliste médicale. Sa thèse portait sur les représentations de l'espace architectural hospitalier et sur la communication entre les acteurs du projet. Ses actuelles thématiques de recherche portent plus particulièrement sur les métiers tournant autour de la maîtrise d'ouvrage (programmateur) et de la maîtrise d'œuvre (étudiants en architecture) : mercierlechine@free.fr 